\title{
Diabetes insipidus: \\ principais aspectos e análise comparativa com diabetes mellitus
}

\author{
Diabetes insipidus: \\ main aspects and comparative analysis with diabetes mellitus
}

\author{
Danielly Mesquita Figueiredo 1; Flávia Lúcia Abreu Rabelo²
}

\section{Resumo}

O diabetes mellitus é uma doença caracterizada pelo excesso de açúcar no sangue e na urina. Os dois tipos mais comuns de diabetes são diabetes mellitus insulino-dependente e diabetes mellitus insulino resistente, e que ambos apresentam comprometimento da regulação da glicemia por ação da insulina. No entanto, existe outra forma de diabetes menos conhecida, mas não menos importante, o diabetes insipidus, que é caracterizado por um distúrbio na síntese, secreção ou ação do ADH (hormônio antidiurético), que pode resultar em síndromes poliúricas com excreção aumentada de urina hipotônica. Fisiologicamente, variações na pressão osmótica ativam osmorreceptores que estimulam a secreção do ADH e esse aumenta a reabsorção de água nos túbulos coletores renais. O presente artigo propõe revisar um estudo abrangente do diabetes insipidus, visando a uma análise comparativa da incidência, diagnóstico, causas, tipos, tratamento e conseqüências entre diabetes insipidus e diabetes mellitus. Diabetes mellitus e insipidus são duas patologias diferentes com a única semelhança que é o próprio diabetes, isto é, poliúria estabelecida. O conhecimento das significativas diferenças entre as patologias estudadas é importante, uma vez que o diabetes insipidus é menos conhecido, porém pode levar a sérias complicações se não for adequadamente tratado.

Palavras-chave: Diabetes. Insipidus. Mellitus. ADH.

\begin{abstract}
Diabetes mellitus is a disease characterized by the excess of sugar in the blood and urine. The two most common types of diabetes are insulin-dependent diabetes mellitus and insulin-resistant diabetes mellitus, both presenting glycemic regulation-damage caused by insulin. Nevertheless, there is another type of diabetes that is less known but not less important, the diabetes insipidus, which is characterized by a problem with the synthesis, secretion or action of the ADH (anti-diuretic hormone) that can result in polyuric syndromes with increased excretion of hypotonic urine. Physiologically, variations in the osmotic pressure activate osmoceptors that stimulate the $\mathrm{ADH}$ secretion, increasing water reabsorption in the kidney collection tubes. This article intends to revise a wide-ranging study on diabetes insipidus, aiming at a comparative analysis of the incidence, diagnosis, causes, types, treatment and consequences between diabetes insidipus and diabetes mellitus. Diabetes mellitus and insipidus are two different pathologies with a single similarity that is the diabetes itself, that is, the polyuria established. The knowledge of the significant differences between the pathologies studied is important once diabetes insipidus is less known, but can lead to serious complications if not properly treated.
\end{abstract}

Key words: Diabetes. Insipidus. Mellitus. ADH.

1 Bióloga. Universidade Federal de Lavras. Departamento de Ciência de Alimentos. Lavras - MG. Cep:37200-000. E-mail: daniellymesquita@gmail.com.

2 Doutora em Bioquímica e Imunologia. Universidade Vale do Rio Doce. Faculdade de Ciências da Saúde - FACS. Governador Valadares - MG. CEP: 35020-220. E-mail: flaviarabelo@univale.br. 


\section{Introdução}

O diabetes é uma doença caracterizada pelo excesso de açúcar no sangue (glicemia) e na urina (glicosúria). Pode ser classificada de acordo com sua patogênese, em diabetes mellitus tipo 1 ou insulinadependente, diabetes mellitus tipo 2 ou insulinaindependente, diabetes gestacional e diabetes insipidus (HALPERN, 2000; LENHINGER; NELSON; COX, 2000).

O diabetes mellitus tipo 1 é uma doença metabólica,crônica,causada pela deficiência ou ação inadequada de insulina. Em pacientes com diabetes tipo 1 sintomáticos, é comum a poliúria, polifagia, polidipsia, perda de peso e alterações visuais. Os pacientes podem sofrer complicações crônicas como aterosclerose, infarto do miorcárdio, além de se tornarem mais susceptíveis a infecções como carbúnculos e furunculose generalizada (GAW et al., 2001; LENHINGER; NELSON; COX, 2000 ).

O diabetes mellitus tipo 2 é um distúrbio comum em que o fator hereditário e a obesidade apresentam importância maior que no diabetes mellitus tipo 1 . Os pacientes diabéticos tipo 2 produzem insulina normalmente, porém suas células são incapazes de usar toda a insulina secretada pelo pâncreas, fazendo com que os níveis de insulina permaneçam altos no sangue, esse fenômeno é conhecido como resistência à insulina. Os diabéticos tipo 2 também apresentam poliúria, polidipsia e polifagia, além de alterações visuais e feridas de difícil cicatrização (GAW et al., 2001).

$\mathrm{O}$ diabetes mellitus gestacional inicia quando o corpo não consegue energia suficiente para a gravidez. Sem insulina suficiente e com concentração de hormônio do crescimento elevado determinando resistência periférica à glicose e conseqüente hiperglicemia, a glicose não consegue sair do sangue e transformar-se em energia. Esta se instala mais comumente em gestantes que ganham muito peso durante a gravidez e poderá acarretar complicações tanto para a mãe como para o feto (CAMPOS, 2004; HALPERN, 2000).
O diabetes insipidus (DI) é caracterizado por um distúrbio na síntese, secreção ou ação do $\mathrm{ADH}$ (hormônio anti-diurético), que podem resultar em síndromes poliúricas, em que ocorre excreção aumentada de urina hipotônica, resultante da ingestão excessiva de água, ou alterações nos canais de aqüaporina-2 $\left(\mathrm{AQP}_{2}\right)$. A hiponatremia pode ocorrer por depleção de sal, mecanismos dilucionais ou metabólicos (NAVES et al., 2003).

Os mecanismos envolvidos na resistência periférica à ação do $\mathrm{ADH}$ levam ao diabetes insipidus nefrogênica, já aqueles relacionados à síntese e secreção do hormônio levam ao diabetes insipidus central ou neurogênico (NAVES et al., 2003).

O diabetes insipidus gestacional é um caso raro, e nela, durante a gravidez, ocorre uma excessiva degradação do $\mathrm{ADH}$ por uma vasopressinase produzida pela placenta (NAVES et al., 2003).

Considerando o conhecimento pouco difundido sobre o diabetes insipidus, é bastante relevante a importância de um estudo da doença, uma vez que a mesma pode trazer muitas complicações ao indivíduo se não for devidamente tratada. Seu diagnóstico correto é imprescindível para o tratamento, portanto se o medicamento prescrito não for específico, a farmacoterapia não alcançará êxito, além de poder agravar o quadro clínico do paciente.

A escassez de material bibliográfico disponível acerca dos vários aspectos do diabetes insipidus, incluindo patogênese, sintomas, diagnóstico, tratamento, prognóstico e incidência, justifica a importância desse trabalho. Além disso, torna-se importante traçar um paralelo entre os principais aspectos dos diferentes tipos de diabetes, com o objetivo de possibilitar uma distinção mais clara entre essas patologias, evitando, assim, diagnósticos e tratamentos inadequados, principalmente em relação ao diabetes insipidus.

O presente artigo propõe revisar um estudo abrangente sobre o diabetes insipidus, enfatizando 
suas causas, tipos, conseqüências, diagnóstico diferencial, complicações e incidência da doença. Do mesmo modo, faz-se uma análise paralela entre os principais aspectos do diabetes insipidus e dos outros tipos de diabetes.

\section{Diabetes insipidus (DI)}

A neurohipófise ou hipófise posterior integra um sistema conhecido como hipotálamo-hipofisário que produz a vasopressina ou hormônio antidiurético $(\mathrm{ADH})$. O diabetes insipidus é uma condição incomum na qual a urina não é concentrada, e sim diluída ou semelhante à água. A concentração urinária que ocorre em pessoas normais é devida à secreção desse hormônio antidiurético (ADH) pela porção posterior da glândula hipofisária e pela ação deste hormônio nos rins, onde ocorre à concentração urinária e o déficit do ADH causa o diabetes insipidus (CAMPOS, 2003; SANCHEZ, 1983).

O diabetes insipidus (DI) é caracterizado pela emissão de grandes volumes de urina (poliúria), abundante ingestão de líquidos, devido à sede constante (polidipsia) e insaciável. Causa sintomas como nictúria (aumento de excreção urinária à noite), ou enurese (excreção involuntária de urina durante o sono). A micção se vê incrementada devido à urina não se encontrar em sua concentração normal. Conseqüentemente, em vez de apresentar coloração amarela, esta se apresenta de cor pálida, incolor e de aparência aquosa, e sua concentração plasmática de sódio é baixa (hiponatremia) (JAKOBY, 2003; ROBERTSON, 2003).

O diabetes insipidus se caracteriza pela geração de grandes volumes de urina diluída. Os mecanismos dessa enfermidade são:

1. Quantidade insuficiente de vasopressina: diabetes insipidus central ou neurogênico.

2. Falha da resposta renal à vasopressina circulante: diabetes insipidus nefrogênico.

As perdas de grandes volumes de urina diluída levam a uma desidratação celular e extracelular, e isso faz com que esta enfermidade se associe à polidipsia e a elevada ingestão de líquido.

\section{Causas e sintomas}

O diabetes insipidus engloba formas diferentes, na qual cada uma tem uma causa específica, por isso elas devem ser tratadas de forma individual. Os principais sintomas são a poliúria e polidipsia, com preferência por líquidos gelados, de início habitualmente abrupto na DI central. Já a nictúria está quase sempre presente nos pacientes com DI central e nefrogênica, mas geralmente não é observada nos casos de polidipsia primária ou psicógena (NAVES et al., 2003). A forma mais comum é causada por uma deficiência de vasopressina ou hormônio antidiurético (ADH), que habitualmente atua sobre os rins, diminuindo a excreção urinária ao incrementar a concentração de urina. Esse tipo de diabetes insipidus se deve a destruição da parte posterior da hipófise, local de armazenamento de vasopressina. Por isso, é comumente chamado de diabetes insipidus hipofisário. Também é conhecido como DI central ou neurogênico. A hipófise posterior pode ser destruída por uma variedade de transtornos secundários incluindo tumores, infecções, traumatismos cranianos, infiltrações, enfermidades autoimunes e vários fatores hereditários. Estes últimos podem ser identificados devido à presença de DI em crianças cujos antecedentes (pais, familiares) apresentem a mesma desordem (BERNALMIZRACHI, 2003).

Uma deficiência primária de $\mathrm{ADH}$ durante a gravidez e resulta uma excessiva degradação desse hormônio por uma vasopressinase produzida pela placenta, é chamado diabetes insipidus gestacional (NAVES et al., 2003). Um outro tipo de DI é causado pela incapacidade de resposta renal frente ao efeito antidiurético de quantidades normais de vasopressina. Esse tipo de DI denomina-se diabetes insipidus nefrogênico, e pode originarse a partir do uso de diferentes medicamentos, drogas, ou de enfermidades renais que incluem 
defeitos genéticos hereditários como mutação recessiva ligada ao cromossomo $\mathrm{X}$ no receptor da vasopressina (GUYTON; HALL, 2002; KOREN, 2003; NGUYEN; NIELSEN; KURTZ, 2003).

Outra forma de DI ocorre quando o efeito da vasopressina é inibido pela ingestão abundante de líquidos. É denominada polidipsia primária, e, na maioria das vezes é causada por uma anormalidade no centro regulador da sede, localizada no hipotálamo. Este subtipo é conhecido como diabetes insipidus dipsógeno ou psicógeno $\mathrm{e}$ é muito difícil diferenciá-la da DI hipofisária, dado que ambas podem se originar dos mesmos transtornos cerebrais. Além do mais, muitas das vezes a DI dipsógena tem causa idiopática, e outras enfermidades psiquiátricas (ROBERTSON, 2003).

\section{Tratamento do diabetes insipidus}

O DI hipofisário é permanente e não pode ser curado (AAFP, 2007), mas seus sintomas podem ser completamente controlados por meio de diversas drogas, entre as quais se encontra uma forma modificada da vasopressina conhecida como desmopressina ou dDAVP (AAFP, 2007). Devido ao DI hipofisário estar associado também à carência de outros hormônios de origem hipofisária, às vezes tratamentos para essas anormalidades também são requisitados (BERNAL-MIZRACHI, 2003; ROBERTSON, 2003).

O diabetes insipidus gestacional também é tratado com dDAVP, mas, neste caso, tanto a deficiência do hormônio como o diabetes insipidus desaparecem entre 4 e 6 semanas depois do parto, momento no qual o tratamento com dDAVP é suspenso (ROBERTSON, 2003).

Já o diabetes insipidus nefrogênico não pode ser tratado com dDAVP, e dependendo da causa que o originou, pode não ser curado, suspendendo-se o medicamento em questão, ou tratando a enfermidade de base. Até os dias de hoje, não foi encontrado tratamento algum para a forma hereditária, assim esta permanece por toda a vida. Atualmente existem tratamentos parciais que podem reduzir os sinais e sintomas do DI nefrogênico (KOREN, 2003; NGUYEN; NIELSEN; KURTZ, 2003).

Diuréticos, principalmente tiazídicos e amilorida, além de agentes antiinflamatórios não-esteróides (AINEs), são importantes opções terapêuticas para o tratamento crônico do DI nefrogênico (HASSAN, 1990; ROBERTSON, 1995; ROBERTSON, 2001; VILAR; LEAL, 2001). Muitas vezes, fazse necessária a associação das duas classes de medicamentos. A combinação e a dose ideais devem ser determinadas empiricamente para cada paciente. Geralmente, o tratamento reduzirá o volume urinário em $50 \%$ a $70 \%$ (ROBERTSON, 1995; ROBERTSON, 2001).

Os diuréticos tiazídicos representam a terapia de escolha para o DI nefrogênico. A amilorida na dose de 5 a 20mg/dia (DE MARCO; LIBERMAN, 2002; SINGER; OSTER; FISHMAN, 1997; VILAR; LEAL, 2001) constitui a melhor opção para o tratamento do DI nefrogênico causado pelo lítio (SINGER; OSTER; FISHMAN, 1997; STONE, 1999). Pode também ser útil em outras formas de DI nefrogênico, mas é menos eficaz do que os tiazídicos por induzir uma menor contração do volume extracelular (STONE, 1999). A combinação dos dois diuréticos pode, contudo, propiciar um efeito antipoliúrico aditivo, bem como prevenir ou atenuar a hipocalemia induzida pelos tiazídicos (SINGER; OSTER; FISHMAN, 1997).

Também no DI dipsógeno, o dDAVP diminui a excreção urinária, assim como no DI hipofisário, mas no primeiro (DI dipsógeno) não se anula a sede excessiva e há um aumento do consumo de líquidos. Assim, isto resulta numa intoxicação hídrica, condição que se associa a sintomas como cefaléias, anorexia, náuseas, e uma diminuição anormal da concentração plasmática de sódio (hiponatremia). Por isso e por não contar com os meios adequados para corrigir a sede intensa, até hoje o DI dipsógeno 
não tem tratamento algum, a não ser para certos sintomas conflituosos, como a nictúria, em que se recomendam pequenas doses de dDAVP à noite antes de dormir (ROBERTSON, 2003).

\section{Diabetes mellitus tipo I e tipo II versus diabetes insipidus}

O diabetes mellitus é amplamente reconhecido como a causa habitual de polidipsia e poliúria, a possibilidade de encontrarmos frente a um diabetes insipidus (DI) é passada despercebida. É muito importante ter o conhecimento sobre o diabetes insipidus, pois seu diagnóstico errado ou tardio pode levar a uma desidratação severa com dano cerebral irreversível, inclusive a morte.
Diabetes insipidus não é sinônimo de diabetes mellitus. Ambos possuem características parecidas, como um incremento de excreção urinária associada a uma sede constante. Não obstante, em seus aspectos restantes, incluindo causas e tratamento, são completamente diferentes. Às vezes o diabetes insipidus é chamado de "diabetes da água", para diferenciá-lo do conhecido diabetes mellitus ou “diabetes do açúcar” (ROBERTSON, 2003).

O diabetes mellitus tipo 1 geralmente ocorre em pessoas com menos de trinta anos (Tabela 1), apresentam-se com baixo peso, tem pouca ou nenhuma insulina endógena, inicia-se rapidamente, a cetose é comum, produz anticorpos contra as ilhotas de Langerhans ocorrendo destruição auto-imune das células das ilhotas pancreáticas, é poligênico, há fatores ambientais implicados (vírus e toxinas).

Tabela 1. Diabetes mellitus tipo I e II versus Diabetes insipidus (Adaptado de GAW et al., 2001).

\begin{tabular}{|c|c|c|c|}
\hline Características Clínicas & DMDI (Tipo I) & DMNDI (Tipo II) & DIABETES INSIPIDUS \\
\hline Idade & $<30$ anos & $>40$ anos & Qualquer \\
\hline Peso & Baixo & Normal ou aumentado & Idade \\
\hline Poliúria & Sim & Sim & Baixo \\
\hline Polidipsia & Sim & Sim & $\operatorname{Sim}$ \\
\hline \multicolumn{4}{|l|}{ Etiologia } \\
\hline & $\begin{array}{l}\text { Destruição } \\
\text { auto-imune das } \\
\text { células das ilhotas } \\
\text { pancreáticas }\end{array}$ & $\begin{array}{l}\text { Obscura. Secreção } \\
\text { prejudicada de insulina e } \\
\text { resistência à insulina }\end{array}$ & $\begin{array}{l}\text { Distúrbios na secreção ou ação } \\
\text { do ADH }\end{array}$ \\
\hline Associações genéticas & Poligênico & Forte & Forte \\
\hline Fatores ambientais & $\begin{array}{l}\text { Vírus e toxinas } \\
\text { implicados }\end{array}$ & $\begin{array}{l}\text { Obesidade, inatividade } \\
\text { física }\end{array}$ & Drogas e agentes químicos \\
\hline
\end{tabular}

O diabetes mellitus tipo 2, geralmente ocorre em indivíduos com mais de quarenta anos, que apresentam peso normal ou aumentado. Inicia-se de forma lenta, ocorrendo cetose sob estresse, está presente a insulina endógena, não há produção de anticorpos contra as ilhotas de Langerhans, sendo a secreção de insulina prejudicada com resistência à insulina. Tem uma forte associação genética, e fatores como obesidade e inatividade física complicam a doença. O diagnóstico de ambos (tipo 1 e 2) é feito por meio da análise da urina (glicose, cetonas ) e glicose sangüínea (GAW et al., 2001; HALPERN, 2000). 
$\mathrm{O}$ diabetes insipidus pode ocorrer em qualquer idade, e quando é de origem genética os sintomas geralmente começam aparecer após 1 ano de idade. Ocorre perda de peso, devido à perda de líqüido constante. Ocorre devido à síntese, secreção ou ação inadequada do hormônio antidiurético $(\mathrm{ADH})$. Pode ser adquirida por uso de drogas, medicamentos, distúrbios hidroelétricos (hipercalcemia, hipercalciúria), doenças sistêmicas, tumores, infecções. Fatores genéticos, como defeitos no receptor da vasopressina (V2R) ou no receptor da aqüaporina $\left(\mathrm{AQP}_{2}\right)$, caracterizam o diabetes insipidus herdado. A falta da ingestão de líquidos pode levar à desidratação (NAVES et al., 2003; NGUYEN; NIELSEN; KURTZ, 2003; SHALEV et al., 2004). O diagnóstico é feito pela análise da urina (osmolaridade) e do sangue (concentração), além de tomografia computadorizada e ressonância magnética (ROBERTSON, 2003).

\section{Conclusão}

Pouca relação existe entre diabetes mellitus e insipidus, uma vez que vários de seus aspectos são diferenciados, a começar pela patogênese. $\mathrm{O}$ conhecimento das significativas diferenças entre as patologias estudadas é importante, uma vez que o diabetes insipidus é menos conhecido, porém pode levar a sérias complicações se não for adequadamente tratado.

\section{Referências}

AMERICAN ACADEMY OF FAMILY PHYSICIANS AAFP. Diabetes Insipidus. 2007. Disponível em: <http:// familydoctor.org/online/famdoces/home/articles/048. printerview.html>. Acesso em: 17 abr. 2008.

BERNAL-MIZRACHI, C. Diabetes insipidus central. 2003. Disponível em: <http://www.urac.com>. Acesso em: 21 abr. 2004.

CAMPOS, S. Diabetes insipidus. 2003. Disponível em: $<$ http://www.drashirleydecampos.com.br>. Acesso em: 19 jul. 2004.
Diabetes gestacional. 2004. Disponível em: $<\mathrm{http} / / / \mathrm{www}$. drashirleydecampos.com.br>. Acesso em: 19 de jul. 2004.

DE MARCO, L. A.; LIBERMAN, B. Metabolismo da água e diabetes insípido. In: CUKIERT, A; LIBERMAN, B. (Ed.). Neuroendocrinología clínica e cirúrgica. São Paulo: Lemos; 2002. p. 563-76.

GAW, A.; COWAN, R. A.; O'REALLY, D. S. J.; STEWART, M. J.; SHEPHERD, J. Bioquímica clínica. 2. ed. Rio de Janeiro: Guanabara Koogan, 2001.

GUYTON, A. C.; HALL, J. E. Tratado de fisiologia médica. 10. ed. Rio de Janeiro : Guanabara Koogan, 2002.

HALPERN, A. Diabetes. In: LEONEL, C. Medicina: mitos e verdades. 4. ed. São Paulo: CIP, 2000. Cap. 6, p. $168-175$.

HASSAN, D.; WIJDICKS, E. F. M.; VERMEULEN, M. Hyponatremia is associated with cerebral ischemia in patients with aneurismal subarachnoid hemorrhage. Annals of Neurology, Boston, v. 27, n. 1, p. 106-108, 1990.

JAKOBY, M. Diabetes insipidus. 2003. Disponível em: $<$ http://www.urac.com>. Acesso em: 21 abr. 2004.

KOREN, A. T. Diabetes insipidus nefrogênica. 2003. Disponível em: <http://www.urac.com>. Acesso em: 21 abr. 2004.

LENHINGER, A. L; NELSON, D. L.; COX, M. M. Princípios de bioquímica. 2. ed. São Paulo: SARVIER, 2000.

NAVES, L. A.; VILAR, L.; COSTA, A. C. F.; DOMINGUES, L.; CASULARI, L. A. Distúrbios na Secreção e Ação do Hormônio Antidiurético. Revista Arquivos Brasileiros de Endocrinolia \& Metabologia, Rio de Janeiro, v. 47, n. 1, p. 467-474. 2003. Disponível em: <http://www.scielo.br/pdf/abem/v47n4/a19v47n4. pdf $>$. Acesso em: 21 abr. 2008.

NGUYEN, M. K.; NIELSEN, S., KURTZ, I. Molecular pathogenesis of nephrogenic diabetes insipidus. Clinical and Experimental Nephrology, Tókio, v. 7, n. 1, p. 9-17, 2003.

ROBERTSON, G. L. Diabetes insipidus. Endocrinology metabolism clinics of North America, Philadelphia, v. 24, n. 3, p. 549-552, 1995.

ROBERTSON, G. L. Antidiuretic hormone - Normal and disordered function. Endocrinology Metabolism Clinics of North America, Philadelphia, v. 30, n. 3, p. 671-694, 2001. 
Qué es la Diabetes insipidus ? The Diabetes Insipidus Foundation, Inc. 2003. Disponível em: <http:// www.diabetesinsipidus.org>. Acesso em: 21 abr. 2008.

SANCHEZ, T. R. Patologia de la neurohipofisis: diabetes insipidus. 1983. Disponível em: <http://uninet.edu/tratado/ c050514.html $>$. Acesso em: 19 jul. 2004.

SHALEV, H.; ROMANOVSKY, I.; KNOERS, N. V.; LUPA, S.; LANDAU, D. Bladder function impairment in aquaporin -2 defective nephrogenic diabetes insipidus. Nephrology Dialysisl Transplantation, Oxford, v. 19, n. 3, p. 608-613, 2004.
SINGER, I.; OSTER, J. R.; FISHMAN, L. M. The management of diabetes insipidus in adults. Archives of Internal Medicine, Chicago, n. 157, p. 1293-1301, 1997.

STONE, K. A. Lithium-induced nephrogenic diabetes insipidus. Journal of the American Board of Family Practice, Waltham, v. 12, n. 1, p.43-47, 1999.

VILAR,L.;LEAL,E.Diabetesinsípido: comodiagnosticar e tratar. In: VILAR, L. (Org.). Endocrinologia clínica. 2. ed. Rio de Janeiro: Medsi; 2001. p.103-124. 
